\title{
A Causal Look at Statistical Definitions of Discrimination
}

\author{
Elias Chaibub Neto \\ elias.chaibub.neto@sagebase.org \\ Sage Bionetworks \\ Seattle, WA
}

\begin{abstract}
Predictive parity and error rate balance are both widely accepted and adopted criteria for assessing fairness of classifiers. The realization that these equally reasonable criteria can lead to contradictory results has, nonetheless, generated a lot of debate/controversy, and has motivated the development of mathematical results establishing the impossibility of concomitantly satisfying predictive parity and error rate balance. Here, we investigate these fairness criteria from a causality perspective. By taking into consideration the data generation process giving rise to the observed data, as well as, the data generation process giving rise to the predictions, and assuming faithfulness, we prove that when the base rates differ across the protected groups and there is no perfect separation, then a standard classifier cannot achieve exact predictive parity. (Where, by standard classifier we mean a classifier trained in the usual way, without adopting pre-processing, in-processing, or post-processing fairness techniques.) This result holds in general, irrespective of the data generation process giving rise to the observed data. Furthermore, we show that the amount of disparate mistreatment for the positive predictive value metric is proportional to the difference between the base rates. For the error rate balance, as well as, the closely related equalized odds and equality of opportunity criteria, we show that there are, nonetheless, data generation processes that can still satisfy these criteria when the base rates differ by protected group, and we characterize the conditions under which these criteria hold. We illustrate our results using synthetic data, and with the re-analysis of the COMPAS data.
\end{abstract}

\section{CCS CONCEPTS}

- Mathematics of computing $\rightarrow$ Causal networks; $\bullet$ Machine learning $\rightarrow$ Supervised learning; • Mathematics and computing $\rightarrow$ Probability and statistics.

\section{KEYWORDS}

Causality, classification, fairness

\section{ACM Reference Format:}

Elias Chaibub Neto. 2020. A Causal Look at Statistical Definitions of Discrimination. In Proceedings of the 26th ACM SIGKDD Conference on Knowledge Discovery and Data Mining (KDD '20), August 23-27, 2020, Virtual Event, CA, USA. ACM, New York, NY, USA, 9 pages. https://doi.org/10.1145/3394486. 3403130

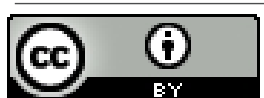

This work is licensed under a Creative Commons Attribution International 4.0 License.

KDD '20, August 23-27, 2020, Virtual Event, CA, USA

(C) 2020 Copyright held by the owner/author(s).

ACM ISBN 978-1-4503-7998-4/20/08.

https://doi.org/10.1145/3394486.3403130

\section{INTRODUCTION}

The notion that a fair classifier should show "predictive parity" (i.e., produce similar positive predictive value for distinct protected groups) is a well established standard in educational and psychological testing [4]. Similarly, the requirement that a classifier should achieve "error rate balance" (i.e., produce similar false positive rates and false negative rates for distinct protected groups) or "equalized odds" (i.e., similar false positive rates and true positive rates for distinct protected groups) or "equality of opportunity" (i.e., similar true positive rates across protected groups) are also widely accepted and adopted criteria $[4,9]$.

A well known impossibility result proved by Chouldechova [4] shows that one needs equal base rates by protected group in order to achieve, at the same time, predictive parity and error rate balance. Corbett-Davies et al [5] independently reached similar conclusions. Kleinberg et al. [11] proved an analogous result where the positive predictive value (PPV) metric is replaced by the calibration requirement, and the error rate balance requirement is replaced by the equality of average scores for the positive and negative classes across the protected groups. See reference [3] for a review of various fairness criteria and their inherent trade-offs.

In this paper, we investigate predictive parity and error rate balance from a causality perspective. We show that standard classifiers cannot achieve exact predictive parity under quite general conditions. Here, we define "standard classifier" as a classifier trained in the usual way, that is, without trying to improve fairness using pre-processing, in-processing, or post-processing techniques [8]. We reserve the term "fair classifier" for classifiers generated using such techniques. We also show that standard classifiers can, on the other hand, achieve error rate balance (and, consequently, the related notions of equalized odds and equality of opportunity), and we present a characterization of data generating processes where standard classifiers can achieve exact error rate balance.

Under the causality framework, the assumptions about the data generation process giving rise to the observed data (namely, the protected variable, the response, and the features) are represented by a causal diagram denoted the "observed data causal diagram". By interpreting the predictions from a machine learning classifier as the output of a well defined data generation process, we can also represent this process using a simple causal diagram, denoted the "prediction causal diagram". Together, these two diagrams provide a full causal representation of a classification task, and, under the faithfulness assumption $[15,20]$, we can use the marginal and conditional independence relations implied by the diagram to: (i) show that it is possible to achieve error rate balance for data generation processes where the outcome variable d-separates [15] the predicted outcome from the protected variable; and (ii) to show that it is impossible to obtain exact predictive parity when base rates differ across the protected groups and there is no perfect separation 
in the data. (Additionally, we show that the amount of disparate mistreatment ${ }^{1}$ for the positive predictive value metric is proportional to the gap between the base rates.) Quite interestingly, the results for the predictive parity criterion hold irrespective of the structure of the observed data causal diagram, and therefore hold in general.

Experiments run with synthetic data sets provide empirical confirmation of our results. Furthermore, a re-analysis of the COMPAS data [13] provides some evidence for the lack of predictive parity in the decile risk scores generated by the COMPAS risk assessment instrument.

The rest of this paper is organized as follows. Section 2 presents relation to previous work, and clarifies that our contribution differs from other works in the literature in two fronts. First, rather than trying to leverage causality in order to develop new causality-based fairness criteria, we use the conditional independence relations implied by the causal model describing a classification task in order to generate new insights about the behavior of widely adopted observational definitions of fairness. Second, our work presents a new impossibility result in fairness (as we show that predictive parity in itself is unachievable when base rates differ by protected group, whereas previous works have established the impossibility of concomitantly satisfying predictive parity and error rate balance under this condition).

Section 3 defines notation and presents key definitions on causality and fairness, which are used in the remaining of the paper. Section 4 shows how base rates, predictive parity, and error rate balance, can be described in terms of conditional independence relations between the outcomes, predicted outcomes and protected variables, whereas Section 5 describes a classification task in terms of causal models. (Together, Sections 4 and 5 provide a bridge between observational definitions of fairness and causal models.) Section 6 presents the main contributions of this paper, namely, Results 1, 2, and 3. Section 7 describes the faithfulness assumption in more detail, as it represents an important assumption in our results and can be sometimes violated in practice. Section 8 presents simulation results illustrating Results 1,2, and 3, as well as, violations of the faithfulness assumption. Section 9 presents a re-analysis of the COMPAS [13] data. Finally, in Section 10 we discuss the implications of our results.

\section{RELATION TO PREVIOUS WORK}

In addition to the literature on the inherent trade-offs of fairness measures (reviewed above), the use of causal reasoning in fairness research has attracted considerable attention in the community. Kilbertus et al. [10] articulated the need to shift fairness research from the search for the "best" observational criteria to paying attention to the causal mechanisms giving rise to the observed data. Kusner et al. [12] proposed the notion of counterfactual fairness, and provided a practical implementation of their approach. Zhang et al. [23] adopted an intervention based approach to model direct versus indirect discrimination as path-specific effects. Nabi and Shpitser [14] adopted a mediation based counterfactual approach to

${ }^{1}$ For any given metric, the amount of disparate mistreatment is defined as the difference in the metric values between the protected groups [22]. quantify path-specific effects, and employ a constrained optimization approach that minimizes discriminatory path specific effects in order to improve fairness. Zhang and Bareinboim [24] adopted a mediation based counterfactual approach to quantify not only direct and indirect path-specific effects, but also spurious effects. Zhang and Bareinboim [25] extended their previous work introducing a new set of counterfactual measures for explaining misclassification disparities, and developed an equality of opportunity classifier leveraging these measures.

A common theme of these contributions is the use of causality to develop more intuitive causality-based measures of discrimination and practical implementations of fair algorithms. These approaches, nonetheless, operate at the intervention or counterfactual levels (layers 2 and 3) of Pearl's ladder of causality "taxonomy" [16, 17]. Our work, on the other hand, is restricted to the association level (layer 1), and uses the conditional independence relations spanned by the causal models underlying a classification task to better understand the behavior of observational definitions of discrimination. Contrary to the previous literature on causality-based fairness, here we do not employ causal inference techniques to improve fairness according to a pre-established fairness definition.

Our work also differs fundamentally from previous work on the trade-offs and impossibility of concomitantly satisfying different notions of fairness. In particular, while the work by Chouldechova [4] and others $[5,11]$ has shown the impossibility of achieving predictive parity and error rate balance when the base rates differ across the protected groups, here we show that predictive parity in itself is unachievable under this circumstance.

\section{PRELIMINARIES}

\subsection{Notation}

Throughout the text we reserve the terms $X, Y$, and $A$ to represent the features, outcome, and protected variables, respectively. For simplicity, we assume that $A$ is binary. We use the subscripts $t r$ and ts to represent, respectively, the training and test sets, and use the notation $X_{t r}, Y_{t r}$, and $A_{t r}$ and $X_{t s}, Y_{t s}$, and $A_{t s}$ to represent the fact that the same random variables $X, Y$, and $A$ generate the data that will be assigned to training and test sets. We reserve the term $\hat{R}_{t s}$ to represent the prediction score generated by a probabilistic classifier (i.e, the predicted probability that a test example belongs to the positive class), while the term $\hat{Y}_{t s}$ is used to represent the respective binarized prediction. We represent marginal independence between two random variables $Z_{1}$ and $Z_{2}$ by $Z_{1} \Perp Z_{2}$, and conditional independence of $Z_{1}$ and $Z_{2}$ given $Z_{3}$ by $Z_{1} \Perp Z_{2} \mid Z_{3}$. The analogous statistical dependence statements are represented by $Z_{1} \not \Perp Z_{2}$ and $Z_{1} \not \Perp Z_{2} \mid Z_{3}$. We use boldface to represent sets of random variables.

\subsection{Causality background}

Following Pearl (2000), we adopt a mechanism-based approach to causation. In this framework, the statistical information encoded in the joint probability distribution of a set of variables $Z=\left\{Z_{1}, \ldots, Z_{p}\right\}$ is supplemented by a directed acyclic graph(DAG) describing our qualitative assumptions about the causal relation between the variables. The joint probability distribution over $Z$ factorizes according to the causal DAG structure, $P\left(Z_{1}, \ldots, Z_{p}\right)=$ 
$\prod_{j} P\left(Z_{j} \mid p a\left(Z_{j}\right)\right)$, where each element, $P\left(Z_{j} \mid p a\left(Z_{j}\right)\right)$, represents an autonomous mechanism describing the relationship between variable $Z_{j}$ and its parents, $p a\left(Z_{j}\right)$. A non-parametric representation of these elements is given by the set o structural causal models $Z_{j}=f_{Z_{j}}\left(p a\left(Z_{j}\right), U_{Z_{j}}\right)$, where $f_{Z_{j}}$ represents a deterministic function of the parents of $Z_{j}$ and of a random disturbance term $U_{Z_{j}}$ (and where the $U_{Z_{j}}$ error terms are independent of each other).

We define a path as any unbroken, nonintersecting sequence of edges in a DAG, which may go along or against the direction of the arrows. We say that a path $\mathcal{P}$ is $d$-separated [15] by a set of nodes $W$ if and only if: (i) $\mathcal{P}$ contains a chain $Z_{j} \rightarrow Z_{m} \rightarrow Z_{k}$ or a fork $Z_{i} \leftarrow Z_{m} \rightarrow Z_{k}$ such that the middle node $Z_{m}$ is in $W$; or (ii) $\mathcal{P}$ contains an inverted fork (or collider) $Z_{j} \rightarrow Z_{m} \leftarrow Z_{k}$ such that the middle node $Z_{m}$ is not in $W$ and no descendant of $Z_{m}$ is in $W$. We say that a set $W$ d-separates a set $Z_{1}$ from a set $Z_{2}$ if and only if $W$ blocks every path from a node in $Z_{1}$ to a node in $Z_{2}$. We say that two variables (or sets of variables) are d-connected if they are not d-separated. Finally, we say that a joint probability distribution over a set of variables is faithful $[15,20]$ to a DAG representing the causal relationships between these variables if no conditional independence relations, other than the ones implied by the d-separation criterion are present.

\subsection{Background on classification performance metrics and fairness related concepts}

The conditional positive predictive value for protected group $a$ is defined as,

$$
P P V_{a}=P\left(Y_{t s}=1 \mid A_{t s}=a, \hat{Y}_{t s}=1\right) .
$$

We say that the predictive parity requirement [4] is satisfied when $P P V_{0}=P P V_{1}$. Conversely, we say that a classifier shows disparate mistreatment [22] for the PPV metric when $P P V_{0} \neq P P V_{1}$. We quantify the amount of disparate mistreatment by $D_{P P V}=P P V_{1}-$ $P P V_{0}$. Similarly, the conditional false positive rate and conditional false negative rate metrics are defined, respectively as,

$$
\begin{aligned}
& F P R_{a}=P\left(\hat{Y}_{t s}=1 \mid A_{t s}=a, Y_{t s}=0\right), \\
& F N R_{a}=P\left(\hat{Y}_{t s}=0 \mid A_{t s}=a, Y_{t s}=1\right) .
\end{aligned}
$$

The error rate balance requirement [4] is satisfied when $F P R_{0}=$ $F P R_{1}$ and $F N R_{0}=F N R_{1}$. Conversely, we say that a classifier shows disparate mistreatment for the FPR (FNR) metric when $F P R_{0} \neq F P R_{1}$ $\left(F N R_{0} \neq F N R_{1}\right)$. Again, we quantify disparate mistreatment by $D_{F P R}=F P R_{1}-F P R_{0}$ and $D_{F N R}=F N R_{1}-F N R_{0}$.

The error rate balance requirement is closely related to the equalized odds and equality of opportunity notions of fairness [9]. The equalized odds notion replaces $F N R_{a}$ by the conditional true positive rate, $T P R_{a}=1-F N R_{a}$, and requires that $T P R_{0}=T P R_{1}$ and $F P R_{0}=F P R_{1}$. The equality of opportunity notion, on the other hand, only requires that $T P R_{0}=T P R_{1}$. Clearly, if a classifier achieves error rate balance, it also achieves equalized odds and equality of opportunity. (Hence, the results that we will present in Section 6 for error rate balance also apply to these other criteria.)

The base rate of each protected group $a$ is defined as,

$$
p_{a}=P\left(Y_{t s}=1 \mid A_{t s}=a\right),
$$

and we say that the base rates differ across the protected groups whenever $p_{0} \neq p_{1}$.
We use the term perfect separation to denote the case where the feature values associated with the outcome values can be completely separated, so that the probability of membership to a given outcome class is either 1 or 0 , and perfect classification is possible.

\section{EXPRESSING FAIRNESS CONCEPTS IN TERMS OF CONDITIONAL INDEPENDENCIES}

Since in this paper we investigate notions of disparate mistreatment from a causality point of view, it is more convenient to express fairness related concepts in terms of statistical dependencies, so that we can use the d-separation criterion [15] to directly read out fairness statements from causal diagrams.

From the definition of conditional independence, we can reexpress the statement of distinct base rates across the protected groups as follows,

$$
p_{0} \neq p_{1} \Leftrightarrow Y_{t s} \not h A_{t s} .
$$

Similarly, we can reexpress the statement that a classifier shows disparate mistreatment for the PPV metric as,

$$
Y_{t s} \not h A_{t s} \mid \hat{Y}_{t s} \Leftrightarrow P P V_{0} \neq P P V_{1},
$$

and the statement of absence of disparate mistreatment for the FPR and FNR metrics as,

$$
\hat{Y}_{t s} \Perp A_{t s} \mid Y_{t s} \Leftrightarrow\left\{\begin{array}{l}
F P R_{0}=F P R_{1}, \\
F N R_{0}=F R R_{1} .
\end{array}\right.
$$

\section{A CAUSAL DIAGRAM REPRESENTATION OF A CLASSIFICATION TASK}

In this section, we describe how the prediction score $\left(\hat{R}_{t s}\right)$ and the respective predicted labels $\left(\hat{Y}_{t s}\right)$, generated by a standard classifier, can be interpreted as the output of structural causal models [15]. This interpretation allows us to use a causal diagram to represent the data generation process giving rise to the predictions.

Training a probabilistic classifier usually corresponds to creating a model, $M$, which corresponds to a (possibly stochastic) function, $g_{1}$, of the training data, $M=g_{1}\left(Y_{t r}, X_{t r}\right)$. The predictor, $\hat{R}_{t s}$, is generated by applying model $M$ to the test set features. In other words, $\hat{R}_{t s}$ usually corresponds to a deterministic function $\hat{R}_{t s}=g_{2}\left(M, X_{t s}\right)$. For instance, in the logistic regression model, $g_{2}$ corresponds to the logistic function. To simplify notation, we represent the predictor by $\hat{R}_{t s}=f_{\hat{R}}\left(Y_{t r}, X_{t r}, X_{t s}\right)$, where $f_{\hat{R}}=g_{2}\left(g_{1}\left(Y_{t r}, X_{t r}\right), X_{t s}\right)$ represents a partial composition of the $g_{1}$ and $g_{2}$ functions. Finally, the predicted test set label usually corresponds to the output of a deterministic function of the predictor $\hat{R}_{t s}$ and a threshold, $t$, defined as, $\hat{Y}_{t s}=f_{\hat{Y}}\left(\hat{R}_{t s}, t\right)$, and assuming value 1 if $\hat{R}_{t s}>t$, and 0 otherwise. For non-probabilistic classifiers, $g_{2}$ directly generates the predicted labels and we have that $\hat{Y}_{t s}=f_{\hat{Y}}\left(Y_{t r}, X_{t r}, X_{t s}\right)$. Note that we do not include the protected variable as a feature in the classifier.

A causal diagram representation of the data generating process giving rise to $\hat{R}_{t s}$ and $\hat{Y}_{t s}$ is presented in Figure 1. Panel a shows the full diagram, while panel b shows a simplified version (that we use in the remaining of the paper). Note that this data generation process is always the same independent of the model $M$ adopted (i.e., $M$ could be a random forest, a logistic regression, and etc). 
Observe, as well, that while we do not explicitly show the threshold node in the simplified diagrams, it is important to keep in mind that distribution of $\hat{Y}_{t s}$ depends on this threshold. Finally, note that in the non-probabilistic case the node $\hat{R}_{t s}$ is removed from the diagram.
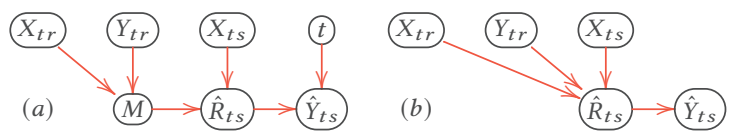

Figure 1: Causal diagram representation of the prediction data generation process.

Figure 2 presents one example of a full causal diagram of the data generation process together with the generation process for the predictions. Observe that the sub-diagrams for the observed training and test set data are identical, but still separated from each other. This encodes the assumption that the observed data points originate from the same data generating process, but independently from each other.

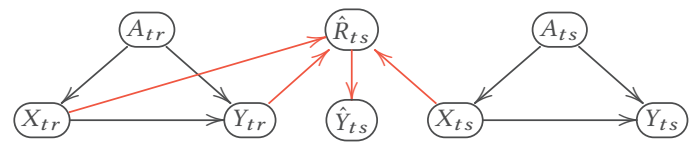

Figure 2: Full causal diagram of a classification problem. The black arrows represent the causal assumptions about the data generation process giving rise to the observed data. The red arrows represent the (known) data generation process generating the predictions.

Figure 3 presents simplified versions of 15 distinct examples of standard classifiers, where the training data is not shown (note that panel a actually corresponds to the causal model in Figure 2). Figure 4, on the other hand, shows a few examples of trivial standard classifiers where $Y$ is marginally independent of $X$. Clearly, the predictive performance of a trivial standard classifier cannot be better than random change, and these classifiers have no practical relevance.

\section{PREDICTIVE PARITY AND ERROR RATE BALANCE FROM A CAUSALITY PERSPECTIVE}

We start with the impossibility result for predictive parity.

RESULT 1. If the outcome and protected variables are associated (i.e., the base rates differ across the protected groups), the faithfulness assumption holds, and there is no perfect separation, then any nontrivial standard classifier will show disparate mistreatment for the $P P V$ metric. In other words, $Y_{t s} \not 1 A_{t s} \Rightarrow P P V_{0} \neq P P V_{1}$.

Proof. From the causal diagram representation of a classifier built with data generated by any arbitrary observed data generation process we have that,

$$
Y_{t s} \not h A_{t s} \Rightarrow Y_{t s} \not h A_{t s} \mid \hat{Y}_{t s},
$$

since $\hat{Y}_{t s}$ does not d-separate $Y_{t s}$ from $A_{t s}$ in the full causal diagram of a non-trivial standard classifier. (Note that the prediction $\hat{Y}_{t s}$
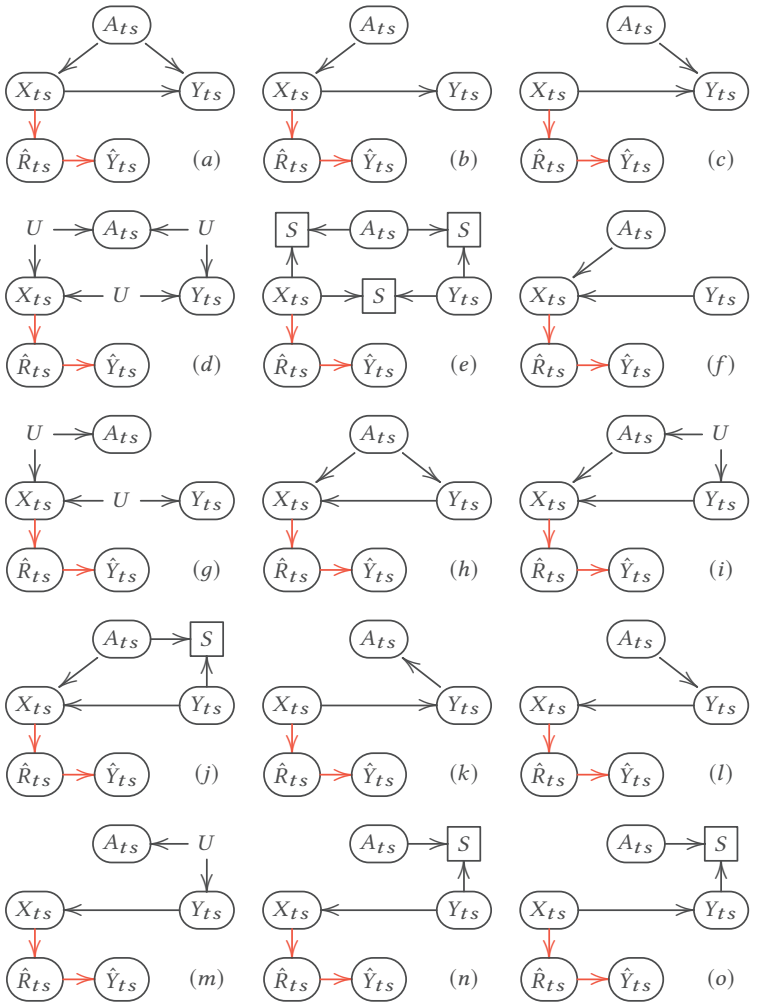

Figure 3: A (non-exhaustive) list of 15 examples of distinct non-trivial standard classifiers. Here, $U$ and $S$ represent unmeasured confounders and selection variables, respectively. The box around $S$ indicates that we are conditioning on $S$.
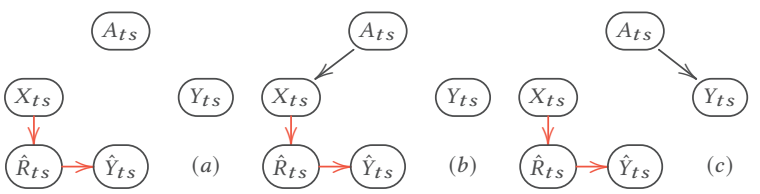

Figure 4: Examples of trivial standard classifiers.

is always generated after the observed data is collected. Hence, it can never be a common cause of $Y_{t s}$ and $A_{t s}$ or a mediator of these variables. Therefore, conditioning on $\hat{Y}_{t s}$ can never d-separate $Y_{t s}$ from $A_{t s}$ in any arbitrary observed data causal diagram.) Then, assuming faithfulness, the result follows from equation (1). (Observe that the result requires the assumption of perfect separation, since (in this boundary case) we have $P P V_{0}=P P V_{1}=1$ independent of whether the base rates differ or not across the protected groups.)

Note that Result 1 is just an "if-then" result, and cannot be further generalized to an "if and only if" result since $P P V_{0} \neq P P V_{1}$ does not necessarily imply $Y_{t s} \not \ell A_{t s}$. (Note that for panels $f$ and $g$ in Figure 3 , we have that $\hat{Y}_{t s}$ is a descendent of $X_{t s}$, and by conditioning on $\hat{Y}_{t s}$ we open the collider path $A_{t s} \rightarrow X_{t s} \leftarrow Y_{t s}$, so that the conditional independence relation $Y_{t s} \not \ell A_{t s} \mid \hat{Y}_{t s}$ holds, and therefore $P P V_{0} \neq P P V_{1}$. However, for these two panels we still have that $Y_{t s} \Perp A_{t s}$, showing that $P P V_{0} \neq P P V_{1}$ does not imply 
$Y_{t s} \not \Perp A$ A $A_{t s}$.) Observe, as well, that all panels in Figure 3 illustrate the fact that $\hat{Y}_{t s}$ cannot d-separate $Y_{t s}$ from $A_{t s}$ for any arbitrary nontrivial standard classifier. Additionally, note that Result 1 would still hold even if $A$ was included as a feature in the classifier. (Note that even if we include an arrow from $A_{t s}$ to $\hat{R}_{t s}$ in all panels of Figure 3, we still have that $\hat{Y}_{t s}$ does not d-separate $Y_{t s}$ from $A_{t s}$ in these cases too.)

Finally, observe that Result 1 only contemplates non-trivial standard classifiers. For trivial classifiers it is possible to see $P P V_{0}=$ $P P V_{1}$ in situations where the marginal independencies $Y_{t s} \Perp X_{t s}$, $Y_{t s} \Perp A_{t s}$, and $Y_{t s} \Perp \hat{Y}_{t s}$ hold (such as in panels a and b of Figure 4), since in this case $P P V_{0}=P P V_{1}=P\left(Y_{t s}=1\right)$. In situations where $Y_{t s} \Perp X_{t s}$ and $Y_{t s} \Perp \hat{Y}_{t s}$, but $Y_{t s} \not \Perp A_{t s}$ (such as in Figure 4c), we have that the $P P V_{a}$ metrics reduce to the base rate probabilities, i.e., $P\left(Y_{t s}=1 \mid A_{t s}=0\right)=P P V_{0} \neq P P V_{1}=P\left(Y_{t s}=1 \mid A_{t s}=1\right)$.

For the FPR and FNR metrics, on the other hand, there are data generating processes for which a standard classifier will show no disparate mistreatment across the protected groups. The next result characterizes when this is the case.

Result 2. For the FPR and FNR metrics, and assuming that the faithfulness assumption holds, any non-trivial standard classifier will show zero disparate mistreatment if and only if the outcome variable $d$-separates the predicted outcome from the protected variable.

Proof. Assuming that the faithfulness assumption holds, whenever $Y_{t s}$ d-separates $\hat{Y}_{t s}$ from $A_{t s}$ in the full causal diagram we have that $\hat{Y}_{t s} \Perp A_{t s} \mid Y_{t s}$ and the result follows from equation (2).

In other words, whenever $Y_{t s}$ d-separates $\hat{Y}_{t s}$ from $A_{t s}$ a standard classifier can show exact error rate balance (exact equalized odds and exact equality of opportunity). Panels k to o in Figure 3 show 5 examples where the FPR and FNR metrics will show zero disparate mistreatment (since $Y_{t s}$ d-separates $\hat{Y}_{t s}$ from $A_{t s}$ ), whereas panels a to j show 10 examples where the FPR and FNR metrics will not show zero disparate mistreatment (as $Y_{t s}$ does not d-separates $\hat{Y}_{t s}$ from $\left.A_{t s}\right)$. Clearly, Result 2 only holds if $A_{t s}$ is not included as a predictor (feature) in the classifier. (Otherwise, it would be impossible to d-separate $A_{t s}$ from $\hat{Y}_{t s}$.)

Finally, observe that Results 1 and 2 provide a more informed context to interpret the relationship,

$$
\frac{F P R_{a}}{1-F N R_{a}}=\frac{p_{a}}{1-p_{a}} \frac{1-P P V_{a}}{P P V_{a}}
$$

derived by Chouldechova [4]. In particular, they can be used together with eq. (3) to show how the gap between the base rates influences the amount of disparate treatment for the PPV metric (but not necessarily for the FPR and FNR metrics). For instance, from Result 2 we see that whenever error rate balance holds (i.e., $F P R_{1}=F P R_{0}$ and $F N R_{1}=F N R_{0}$ ), it follows from eq. (3) that,

$$
\frac{p_{1}}{1-p_{1}} \frac{1-P P V_{1}}{P P V_{1}}=\frac{p_{0}}{1-p_{0}} \frac{1-P P V_{0}}{P P V_{0}},
$$

so that it is easy to see that the amount of disparate mistreatment for the PPV metric is proportional to the difference between the base rates ${ }^{2}$. As we show next, this result is also true in general, even when error rate balance does not hold.

RESUlt 3. For the PPV metric, the amount of disparate mistreatment is proportional to the difference in base rates.

Proof. We will show first that increases in base rate differences lead to increases in disparate mistreatment.

From equation 2.6 in Chouldechova [4] we have that,

$$
\frac{F P R_{a}}{1-F N R_{a}}=\frac{p_{a}}{1-p_{a}} \frac{1-P P V_{a}}{P P V_{a}}
$$

Now, consider the set of all possible classifiers for which the difference in the ratios $F P R_{a} /\left(1-F N R_{a}\right)$ is constant, that is,

$$
\frac{F P R_{1}}{1-F N R_{1}}-\frac{F P R_{0}}{1-F N R_{0}}=\delta \text {, }
$$

for a fixed $\delta$. Denote this set by $C_{\delta}$. Clearly, $C_{\delta}$ includes classifiers with distinct differences in base rates (i.e., classifiers with distinct $\left.\epsilon=p_{1}-p_{0}\right)$ but that still show a difference $\delta$. Note that $\delta$ will be equal to 0 for classifiers that achieve error rate balance, but will be different from 0 for classifiers that produce disparate mistreatment for the FPR and FNR metrics.

Expressing $\delta$ in terms of the PPV metric we have that,

$$
\frac{p_{1}}{1-p_{1}} \frac{1-P P V_{1}}{P P V_{1}}-\frac{p_{0}}{1-p_{0}} \frac{1-P P V_{0}}{P P V_{0}}=\delta \text {. }
$$

Now, consider a sequence of subsets of $C_{\delta}$ composed of classifiers with increasing base rates for protected group 1 and decreasing base rates for group 0 , that is, subsets with increasing gaps, $\epsilon_{i}=$ $p_{1, i}-p_{0, i}$, for $i=1,2, \ldots$, where $p_{1, i}>p_{1, j}$ and $p_{0, i}<p_{0, j}$, for $i<j^{3}$. Denote these subsets by $C_{\delta, \epsilon_{i}}$.

Note that as we increase the gap between the base rates (i.e., move along the sequence $\left.\left\{C_{\delta, \epsilon_{1}}, C_{\delta, \epsilon_{2}}, \ldots\right\}\right)$, we increase $p_{1, i}$ and decrease $p_{0, i}$. Now, observe that increasing $p_{1, i}$ and decreasing $p_{0, i}$ is equivalent to increasing the ratio $p_{1, i} /\left(1-p_{1, i}\right)$ and decreasing the ratio $p_{0, i} /\left(1-p_{0, i}\right)$. From equation 6 we see that the only way that we can concomitantly increase $p_{1, i} /\left(1-p_{1, i}\right)$ and decrease $p_{0, i} /\left(1-p_{0, i}\right)$, without changing the value of $\delta$, is to concomitantly decrease the ratio $\left(1-P P V_{1, i}\right) / P P V_{1, i}$ and increase the ratio $\left(1-P P V_{0, i}\right) / P P V_{0, i}$. Now, because decreasing the ratio $\left(1-P P V_{1, i}\right) / P P V_{1, i}$ and increasing the ratio $\left(1-P P V_{0, i}\right) / P P V_{0, i}$ is equivalent to increasing $P P V_{1, i}$ and decreasing $P P V_{0, i}$, it follows that increasing the gap between the base rates increases the amount of disparate mistreatment for the PPV metric.

Finally, observe that a similar argument can be used to show that decreases in base rate differences lead to decreases in disparate mistreatment

\footnotetext{
${ }^{2}$ To see why, consider an increase in the gap between the base rates obtained by increasing $p_{1}$ and decreasing $p_{0}$. Note that increasing $p_{1}$ will lead to an increase in the ratio $p_{1} /\left(1-p_{1}\right)$, while decreasing $p_{0}$ will lead to a decrease in the ratio $p_{0} /\left(1-p_{0}\right)$. Now, the only way to concomitantly increase $p_{1}$ and decrease $p_{0}$ while maintaining the equality in eq. (4) is to concomitantly decrease the ratio $\left(1-P P V_{1}\right) / P P V_{1}$ and increase the ratio $\left(1-P P V_{0}\right) / P P V_{0}$. Observe, however, that this last statement is equivalent to increasing $P P V_{1}$ and decreasing $P P V_{0}$. Hence, we can see that an increase in the difference between the base rates leads to an increase in disparate mistreatment. A similar argument shows that a decrease in the base rate gap leads to a decrease in disparate mistreatment.

${ }^{3}$ While we focus on the case where the gap increase is obtained by increasing $p_{1, i}$ and decreasing $p_{0, i}$, the same results hold in the case that we increase $p_{1, i}$ and keep $p_{0, i}$ fixed, or in the case that $p_{1, i}$ is fixed and $p_{0, i}$ decreases.
} 
Now, we explain why a similar result does not hold for the FPR and FNR metrics. Reorganizing eq. (5) as,

$$
\frac{P P V_{a}}{1-P P V_{a}}=\frac{p_{a}}{1-p_{a}} \frac{1-F N R_{a}}{F P R_{a}}
$$

we can consider the set of possible classifiers for which the difference in the ratios $P P V_{a} /\left(1-P P V_{a}\right)$ is constant, that is,

$$
\frac{P P V_{1}}{1-P P V_{1}}-\frac{P P V_{0}}{1-P P V_{0}}=\gamma,
$$

for $\gamma \neq 0$ (note that from Result $1, \gamma$ cannot be equal to 0 when the base rates differ), so that,

$$
\frac{p_{1}}{1-p_{1}} \frac{1-F N R_{1}}{F P R_{1}}-\frac{p_{0}}{1-p_{0}} \frac{1-F N R_{0}}{F P R_{0}}=\gamma .
$$

Similarly to before, consider a sequence of classifiers $C_{\gamma, \epsilon_{i}}$ with increasing gaps $\epsilon_{i}$. Again, observe that as we move along the sequence, we increase $p_{1, i}$ and decrease $p_{0, i}$. Now, note that increasing $p_{1, i}$ and decreasing $p_{0, i}$ is equivalent to increasing the ratio $p_{1, i} /(1-$ $\left.p_{1, i}\right)$ and decreasing the ratio $p_{0, i} /\left(1-p_{0, i}\right)$. From equation 9 we see that the only way that we can concomitantly increase $p_{1, i} /\left(1-p_{1, i}\right)$ and decrease $p_{0, i} /\left(1-p_{0, i}\right)$, without changing the value of $\gamma$, is to concomitantly decrease the ratio $\left(1-F N R_{1, i}\right) / F P R_{1, i}$ and increase the ratio $\left(1-F N R_{0, i}\right) / F P R_{0, i}$.

Now, observe that, contrary to the PPV case where a decrease in $\left(1-P P V_{1, i}\right) / P P V_{1, i}$ necessarily implies an increase in $P P V_{1, i}$ and an increase in $\left(1-P P V_{0, i}\right) / P P V_{0, i}$ necessarily implies a decrease in $P P V_{0, i}$, we now have that a decrease in $\left(1-F N R_{1, i}\right) / F P R_{1, i}$ does not necessarily imply an increase in $F P R_{1, i}$ (for instance, the ratio will still decrease when $F P R_{1, i}$ decreases but $F N R_{1, i}$ increases faster than $F P R_{1, i}$ decreases). Similarly, an increase in the ratio (1$\left.F N R_{0, i}\right) / F P R_{0, i}$ does not necessarily imply a decrease in $F P R_{0, i}$.

Hence, it does not necessarily follows that an increase in the difference between the base rates will lead to an increase in disparate mistreatment for the FPR metric (and, similarly, to the FNR metric).

\section{ON THE FAITHFULNESS ASSUMPTION}

Faithfulness $[15,20]$ is a key assumption in causal analysis. Geometrically, the set of parameterizations of a joint distribution that lead to unfaithfulness issues can be interpreted as a collection of hypersurfaces (representing constraints in the parameter values) relative to the full parameter space formed by a hypercube with dimension equal to the number of parameters [21]. Hence, the main justification for the faithfulness assumption is that the set of unfaithful distributions has probability measure zero (as it corresponds to a set of hypersurfaces in a hypercube). For practical purposes, however, this assumption can still be violated, since some of the parameterizations of the joint distribution can generate distributions that are very close to one (or more) of these "unfaithful hypersurfaces". This issue is particularly exacerbated by sampling noise. Therefore, in practice, some of the conditional independence relations observed in data sampled from such an "approximately unfaithful" distribution might not be implied by the causal diagram [21]. Clearly, the larger the number of variables in the causal diagram, the larger the probability that at least a few of the conditional independence relations implied by the graph will not hold in the data. While this issue is of great concern for causal discovery algorithms (which attempt to learn the full causal structure of the data generating process), it is somewhat less of a problem in our setting, as our results only involve the $Y_{t s}, A_{t s}$, and $\hat{Y}_{t s}$ variables and, therefore, do not really require that all conditional independence relations implied by the causal diagram, across all the variables, hold in the data. For our results, it suffices that faithfulness holds for the conditional independence relations involving these three variables. (Still, the synthetic data experiments that we present in the next section illustrate that a small fraction of the synthetic data sets still showed unfaithful relations among these variables.)

\section{SYNTHETIC DATA ILLUSTRATIONS}

Here, we use synthetic data to illustrate Results 1,2, and 3 presented in Section 6, as well as, to show how faithfulness issues can still sometimes arise in practice. (Note that we do not attempt to perform an extensive simulation study, since the main contribution of the paper are the formal results and these synthetic data experiments are simply meant as illustrations.)

We consider two causal models: Model 1, presented in Figure 5, illustrates the case where $Y_{t s}$ d-separates $A_{t s}$ from $\hat{Y}_{t s}$; Model 2, presented in Figure 2, represents the case where $A_{t s}$ and $\hat{Y}_{t s}$ are still d-connected conditional on $Y_{t s}$.

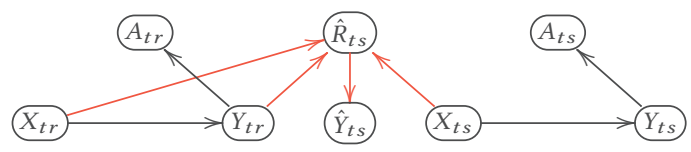

Figure 5: Data generation process for Model 1.

In our simulations, we generated binary $X, A$, and $Y$ variables sampled from the factorized joint distributions,

$$
\begin{gathered}
P(X=x) P(Y=y \mid X=x) P(A=a \mid Y=y), \\
P(A=a) P(X=x \mid A=a) P(Y=y \mid X=x, A=a),
\end{gathered}
$$

for Model 1 and Model 2, respectively.

For each of these models, we simulated 100 synthetic datasets. Each dataset was generated by recursively sampling values from each element of the factorized distribution. For instance, for Model 1 , the factorized distribution is parameterized by 5 parameters (namely, $P(X=1)=\theta_{1}, P(Y=1 \mid X=0)=\theta_{2}, P(Y=1 \mid$ $\left.X=1)=\theta_{3}, P(A=1 \mid Y=0)=\theta_{4}, P(A=1 \mid Y=1)=\theta_{5}\right)$, and we sample a data point, $(X, A, Y)$, as follows:

(1) Randomly sample $\theta_{j}$, for $j=1, \ldots, 5$ in the $[0,1]$ range.

(2) Sample $X$ from a Bernoulli $\left(\theta_{1}\right)$ distribution.

(3) If $X=0$, sample $Y$ from a Bernoulli $\left(\theta_{2}\right)$. Otherwise, if $X=1$, sample $Y$ from a Bernoulli $\left(\theta_{3}\right)$.

(4) If $Y=0$, sample $A$ from a $\operatorname{Bernoulli}\left(\theta_{4}\right)$. Otherwise, if $Y=1$, sample $A$ from a Bernoulli $\left(\theta_{5}\right)$.

In our simulations, rather than directly sampling the $\theta_{j}$ parameter values from independent Uniform $(0,1)$ distributions, we employ an optimized Latin hypercube design [18] to select the $\theta_{j}$ parameter values in the $[0,1]$ range. (In the field of computer experiments, optimized Latin hypercube designs represent a popular choice, as they achieve a more regular sampling of the parameter space than simply sampling each $\theta_{j}$ from a uniform distribution in the $[0,1]$ range.)

For each of the simulated datasets, we: (i) trained a Bayes classifier (using only $X$ as a predictor); (ii) calculated the disparate 
mistreatment values, $D_{P P V}=P P V_{1}-P P V_{0}, D_{F P R}=F P R_{1}-F P R_{0}$, and $D_{F N R}=F N R_{1}-F N R_{0}$; and (iii) recorded the base rate difference, $p_{1}-p_{0}$. In all simulations we adopted 50:50 train/test random splits of the simulated data.

Figure 6 presents the results for simulations based on very large sample sizes $(n=1,000,000)$. Panels a and b show scatter plots of the base rate differences versus $D_{P P V}$ for Models 1 and 2, respectively. Note the strong associations between the base rate difference and the amount of disparate mistreatment. These empirical results not only corroborate the prediction of Result 1 that we cannot observe $D_{P P V}=0$ whenever the base rates differ, but also provide empirical evidence that the amount of disparate mistreatment is proportional to the difference in base rates (Result 3).
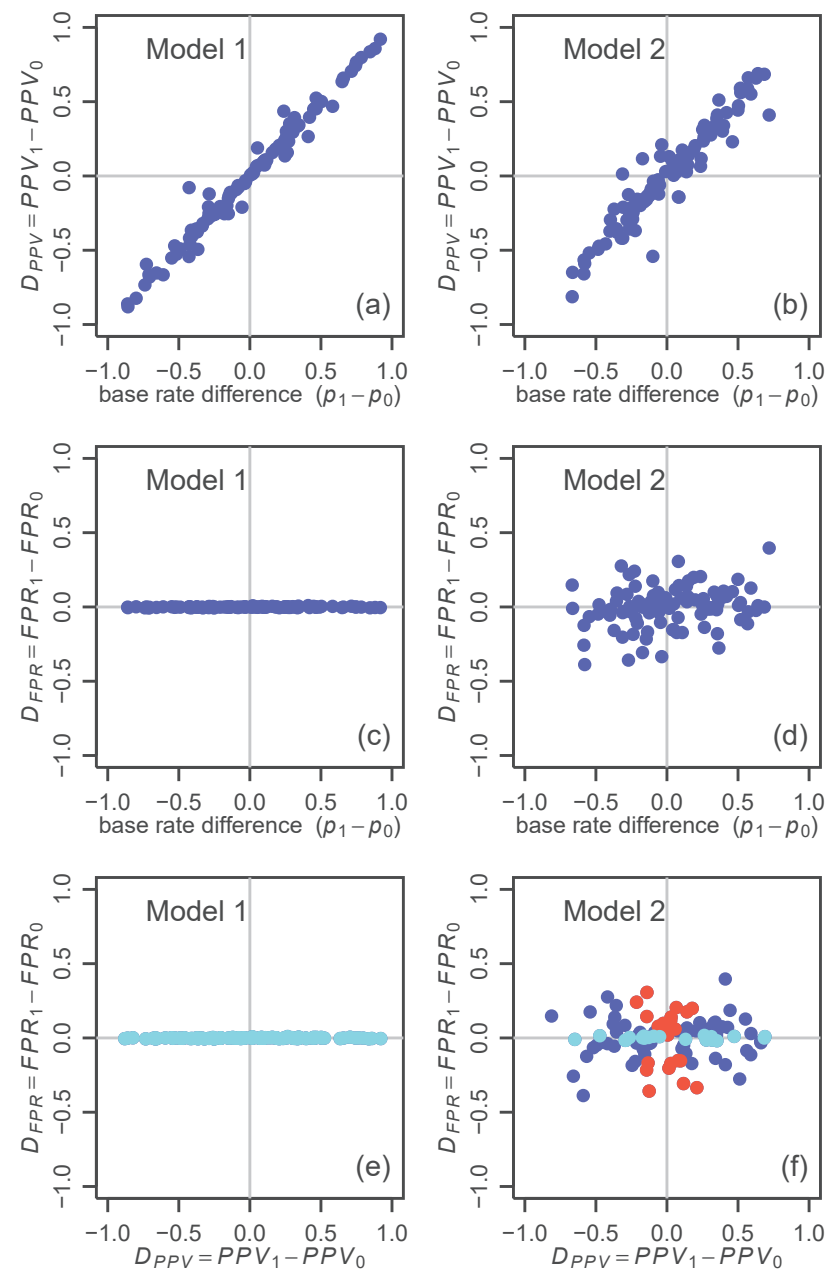

Figure 6: Simulation study results based on $n=1,000,000$.

Panels $\mathrm{c}$ and $\mathrm{d}$ show analogous scatter plots of $p_{1}-p_{0}$ versus $D_{F P R}$, while panels e and $\mathrm{f}$ show scatter plots of $D_{P P V}$ versus the $D_{F P R}$. In accordance with Result 2, data simulated from Model 1 (panels c and e) show negligible $D_{F P R}$ (recall that, in this model, $Y_{t s}$ d-separates $A_{t s}$ from $\hat{Y}_{t s}$ ). Most of the datasets generated from Model 2 (panels $\mathrm{d}$ and $\mathrm{f}$ ), on the other hand, showed non-negligible levels of $D_{F P R}$ (since, in this case, $A_{t s}$ and $\hat{Y}_{t s}$ are still d-connected conditional on $\left.Y_{t s}\right)$.

Panel $\mathrm{f}$ shows that while most of the simulations generated stronger $D_{P P V}$ than $D_{F P R}$ (blue dots), $23 \%$ of the simulations actually showed stronger $D_{F P R}$ than $D_{P P V}$ (red dots). Additionally, note that in $22 \%$ of the simulations (cyan dots) $D_{F P R} \approx 0$, suggesting that the faithfulness assumption has been violated. (Recall that, for Model 2, the d-separation criterion implies that $\hat{Y}_{t s} \not 1 A_{t s} \mid Y_{t s}$. However, the fact that $F P R_{1}-F P R_{0} \approx 0$ shows that the data is actually consistent with the "unfaithful" relation $\hat{Y}_{t s} \Perp A_{t s} \mid Y_{t s}$.) The results for the FNR metric are similar to the FPR results presented above, and are reported in Figure 7 for completeness.
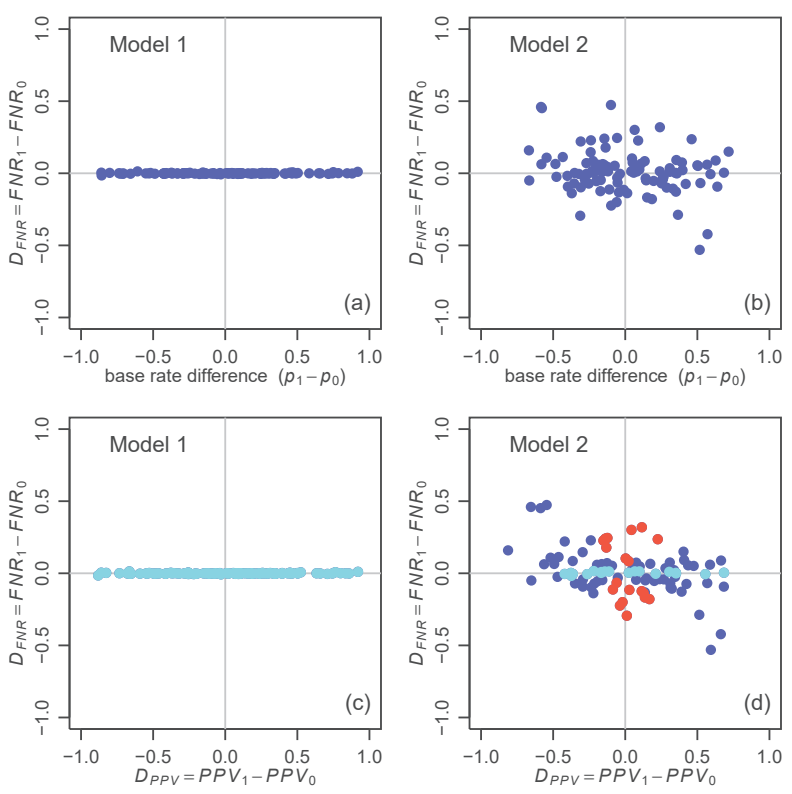

Figure 7: Simulation study results comparing base rate differences $\left(p_{1}-p_{0}\right)$ against $D_{F N R}$, and $D_{P P V}$ against $D_{F N R}$.

\section{ON THE PREDICTIVE PARITY OF THE COMPAS SCORE}

The COMPAS risk assessment instrument [1] has been used as an example where predictive parity with respect to race is (approximately) satisfied [7]. At first sight, this might seem to contradict Result 1 , since the base rates for black and white subjects are given, respectively, by 0.51 and 0.39 in the COMPAS data. Here, we reanalyze this dataset, and show that it actually does not provide a clear example of approximate predictive parity.

Figure 8a, presents the $P P V_{a}$ values for black and white defendants, computed across all 10 possible discretizations of the COMPAS decile score ("high-risk cutoff") ${ }^{4}$. Because the $P P V_{a}$ values of

\footnotetext{
${ }^{4}$ The COMPAS instrument produces a decile score (i.e., a score varying from 1 to 10 ) that can be used to assess the risk of recidivism. In order to compute the $P P V_{a}$, the decile score needs to be first discretized into high-risk and low-risk groups using an (arbitrary) cutoff threshold. For instance, if we let $S$ represent the decile score, and $s_{H R}$ represent the cutoff threshold, then we obtain a high-risk score if $S>s_{H R}$ and a low-risk score if $S \leq s_{H R}$. The $P P V_{a}$ score is then computed as $P\left(Y=1 \mid S>s_{H R}, A=a\right)$. Note that for $s_{H R}=0$ we employ the entire dataset in the estimation of the $P P V_{a}$ scores, but for $s_{H R}=k$, for $k=1, \ldots, 9$, we discard all the subjects with $S \leq k$.
} 
black and white defendants tend to roughly track each other, this figure (which corresponds to Figure 1 of Chouldechova's paper) has been informally used to make the case in favor of (approximate) predictive parity of the COMPAS dataset. The red error bars represent the 95\% confidence intervals of the $P P V_{a}$ estimates. (Note how the error bars overlap for cutoffs greater than or equal to 4 , suggesting that differences in $P P V_{a}$ are not statistically significant.)

However, the fact that the error bars do not overlap for cutoffs less than or equal to 3, provide evidence for the lack of predictive parity. Furthermore, as illustrated in Figure $8 \mathrm{~b}$, the sample sizes used for the estimation of the $P P V_{a}$ scores decrease drastically for larger high-risk cutoffs, suggesting that the perceived predictive parity might be actually due to the lack of statistical power to detect moderate differences in the $P P V_{a}$ estimates. (See the Appendix for more formal power calculations.) Overall, these results suggest that the COMPAS data do not represent a clear example of approximate predictive parity. At least for cutoffs less than or equal to 3 (where the sample sizes are the largest) this data provides empirical evidence for the lack of predictive parity, in accordance with the results from Result 1 (which anticipates lack of predictive parity when the base rates differ by protected group).
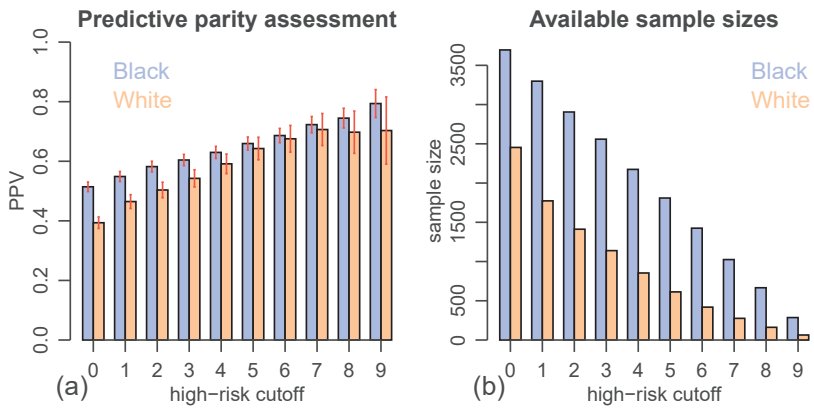

Figure 8: Predictive parity assessment for the COMPAS data.

\section{DISCUSSION}

In this paper, we show that a standard classifier cannot achieve exact predictive parity, under general conditions, and that the amount of disparate mistreatment for the PPV metric is proportional to the gap between the base rates. While these results raise questions about the formal validity of the predictive parity requirement as a fairness criterion, they do not necessarily imply that approximate predictive parity cannot be seen in practice. First, approximate predictive parity can arise simply because the conditional dependence relation $Y_{t s} \not \Perp A_{t s} \mid \hat{Y}_{t s}$ (implied by d-separation) might not hold in the data. As illustrated in our simulations, a few synthetic datasets generated from Model 2 (Figure 2), for which the relation $Y_{t s} \not 1 A_{t s} \mid \hat{Y}_{t s}$ should have held in the data, actually showed the unfaithful relation $Y_{t s} \Perp A_{t s} \mid \hat{Y}_{t s}$ (cyan dots on Figure 6f). Second, Result 1 only shows that it is impossible to achieve exact predictive parity. It does not imply that small (yet real) amounts of disparate mistreatment for the PPV metric cannot be seen in practice. In applications where a small difference in $P P V_{a}$ is negligible for all practical purposes, it might be reasonable to accept approximate predictive parity as an operational definition of fairness.
Furthermore, our results only apply to standard classifiers. They don't say anything about "fair classifiers" generated using preprocessing, in-processing, or post-processing techniques [8]. For instance, in the context of the error rate balance criterion, inprocessing techniques attempting to reduce disparate mistreatment for the FPR and/or FNR metrics [2, 22, 25] employ modified optimization schemes that generate predictions that, conditional on the outcome variable, are less associated with the protected variable (in comparison to a standard classifier). In situations where the data generation process implies that $\hat{Y}_{t s} \not \Perp A_{t s} \mid Y_{t s}$ (i.e., the condition under which Result 2 shows that a non-trivial standard classifier will present disparate mistreatment for the FPR and FNR metrics), these fair classifiers produce solutions that are closer to the unfaithful relation $\hat{Y}_{t s} \Perp A_{t s} \mid Y_{t s}$. (The observation that unfaithfulness emerges naturally when designing fair predictions has also been made before [10].)

However, it is important to point out that while several inprocessing techniques have been proposed to reduce disparate mistreatment for the FPR and/or FNR metrics, we are not aware of any references handling disparate mistreatment for the PPV metric. The only mention on the literature is in Zafar et al. [22] who point out that extending their framework to disparate mistreatment of the FDR metric (and, therefore, to the PPV metric, since FDR = 1 - PPV) is a non-trivial task due to the computational complexities involved. While the lack of practical implementations of fair classifiers targeting PPV do not prove that it cannot be done, it certainly shows it is not a straightforward task. In any case, it is interesting to notice that predictive parity is a problematic criterion for both standard and fair classifiers, while error rate balance and related criterions (such as equalized odds and equality of opportunity) are not only sensible from a formal standpoint but also have lead to practical implementations of fair classifiers.

Finally, we point out that while most of the computer science literature on fairness has focused on the development of fair classifiers, in practice, it is still important to understand the behavior of standard classifiers, since logistic regression and other standard classifiers are often used in the evaluation of the biases of risk assessment tools in the field of criminal justice and behavior [7, 19].

\section{APPENDIX: POWER ANALYSIS FOR THE COMPAS DATA}

As described in Section 9, the red error bars in Figure 7a represent 95\% confidence intervals for the estimated $P P V_{a}$ values. (These confidence intervals are calculated using the standard confidence interval approach for proportions based on the formula, $\hat{p} \pm 1.96 \sqrt{\hat{p}(1-\hat{p}) / n}$, where $\hat{p}$ represents the proportion estimate (i.e., the $P P V_{a}$ estimate in our context), and $n$ represents the sample size available to estimate the proportion.) These confidence intervals can be used to test the null hypothesis of predictive parity (i.e., that PPV scores of blacks and white defendants are equal) against the alternative hypothesis of disparate mistreatment (i.e., that the PPV scores of blacks and white defendants are different). If the error bars do not intersect we can reject the null hypothesis of predictive parity. Figure 7a shows that for $s_{H R} \leq 3$ (where $s_{H R}$ represents the high-risk cutoffs) the confidence intervals do not overlap, and we can reject the null hypothesis. For cutoffs equal or 
greater than 4, on the other hand, we do not have evidence to reject the null hypothesis of predictive parity. We point out, however, that lack of evidence to reject the null is not the same as evidence in favor of the null. For instance, when the sample sizes are small, it is possible that the test fails to reject the null because it lacks statistical power to detect the alternative rather than because the null is true. Since Figure $7 \mathrm{~b}$ shows fairly reduced sample sizes for high cutoffs it is possible that lack of power might explain why the error bars overlap for higher $s_{H R}$ values in Figure 7a.

In order to formally evaluate this hypothesis, we used the method described on reference [6] to compute the statistical power to detect differences in proportions, based on the available sample sizes and observed $P P V_{a}$ values in the COMPAS data. (These calculations, however, should be viewed as approximate, since they assume that the proportions are independent, what might not be the case for the $P P V_{a}$ estimates.) Figure 9a reports the results.
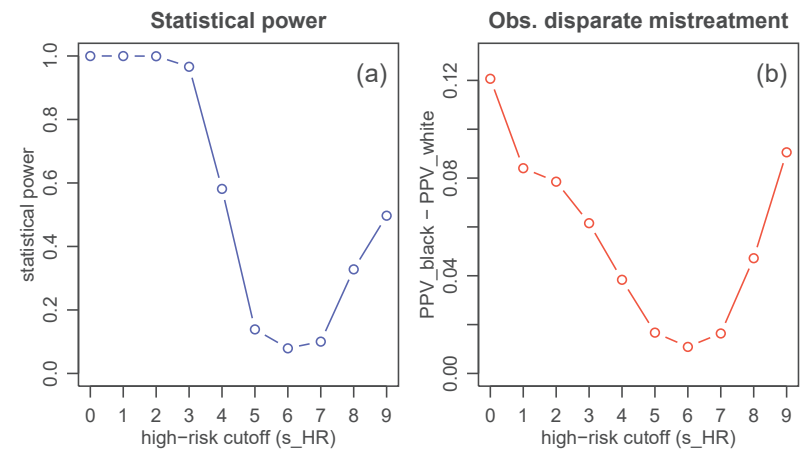

Figure 9: Power analysis for the COMPAS data.

Generally speaking, the power to detect a difference in two proportions will increase with larger differences in the proportions and with larger sample sizes. Panel a reports the power to detect disparate mistreatment at a 0.05 significance level, while panel b shows the observed disparate mistreatment values (i.e., $P P V_{\text {black }}-$ $\left.P P V_{\text {white }}\right)$ for the different cutoffs. As expected, the shape of the curve in panel a mirrors the differences in proportions, $P P V_{\text {black }}-$ $P P V_{\text {white }}$, with larger differences leading to increased power.

For cutoff values between 0 and 3, Figure 9a shows statistical power close to 1 . This shows that we have enough data to detect the large differences in PPV observed at these cutoffs, and reflects the fact that the confidence intervals (error bars) in Figure 7a have small ranges due to the large sample sizes available for their computation.

For cutoff values between 5 and 7 we observe a drastic reduction in power due in most part to the small differences in PPV scores rather than to the reduction in sample sizes (that are still reasonably large for these cutoffs, as shown in Figure 7b, what is also reflected by the small confidence interval amplitudes observed for these cutoffs in Figure 7a). Hence, for cutoffs between 5 and 7 we indeed have evidence in favor of the null hypothesis of predictive parity.

For cutoffs equal or greater than 8 , on the other hand, we observe an increase in power (despite the even smaller sample sizes) due to the larger differences in PPV values. The power, however, is still quite limited for these cutoffs $(<0.6)$, suggesting that the failure to reject the null might indeed be due to the small sample sizes. (Note the much larger ranges of the confidence intervals for these cutoffs in Figure 7a.) Hence, for $s_{H R} \geq 8$ the overlapping confidence intervals should not be interpreted as evidence of predictive parity.

\section{REFERENCES}

[1] Julia Angwin, Jeff Larson, Suria Mattu, and Lauren Kirchner. 2016. Machine bias: There's software used across the country to predict future criminals. And it's biased against blacks. ProPublica, May 23, 2016.

[2] Yahav Bechavod and Katrina Ligget. 2017. Penalizing unfairness in binary classification. arXiv:1707.00044.

[3] Richard Berk, Hoda Heidari, Shahin Jabbari, Michael Kearns, Aaron Roth. 2017. Fairness in criminal justice risk assessment: the state of the art. arXiv:1703.09207.

[4] Alexandra Chouldechova. 2017. Fair prediction with disparate impact: A study of bias in recidivism prediction instruments. Big Data, 5(2):153-163.

[5] Sam Corbett-Davies, Emma Pierson, Avi Feller and Sharad Goel. 2016. A computer program used for bail and sentencing decisions was labeled biased against blacks. Its actually not that clear. Washington Post, October 7, 2016.

[6] Joseph L. Fleiss, Alex Tytun and Hans K. Ury. 1980. A simple approximation for calculating sample sizes for comparing independent proportions. Biometrics, 36:343-6.

[7] Anthony W. Flores, Kristin Bechtel, and Christopher T. Lowenkamp. 2016. False positives, false negatives, and false analyses: A rejoinder to "machine bias: There's software used across the country to predict future criminals. And it's biased against blacks.". Technical report, Crime \& Justice Institute, September 2016.

[8] Sara Hajian and Josep Domingo-Ferrer. 2013. A methodology for direct and indirect discrimination prevention in data mining. IEEE Transactions on Knowledge and Data Engineering, 25(7):1445-1459.

[9] Moritz Hardt, Eric Price, and Nathan Srebro. 2016. Equality of opportunity in supervised learning. Advances in Neural Information Processing Systems, 2016. (NIPS 2016), pp. 3315Ü3323.

[10] Nikki Kilbertus et al. 2017. Avoiding discrimination through causal reasoning. Advances in Neural Information Processing Systems, 2017. (NIPS 2017), pp. 656-666.

[11] Jon Kleinberg, Sendhil Mullainathan, Manish Raghavan. 2016. Inherent trade-offs in the fair determination of risk scores. arXiv:1609.05807.

[12] Matt Kusner, Joshua Loftus, Chris Russell, and Ricardo Silva. 2017. Counterfactual fairness. Advances in Neural Information Processing Systems, 2017. (NIPS 2017).

[13] Jeff Larson, Surya Mattu, Lauren Kirchner and Julia Angwin. 2016. How We Analyzed the COMPAS Recidivism Algorithm. ProPublica, May 23, 2016.

[14] Razieh Nabi, and Ilya Shpitser. 2018. Fair inference on outcomes. The ThirtySecond AAAI Conference on Artificial Intelligence, 2018 (AAAI-18), pp. 1931-1940.

[15] Judea Pearl. 2009. Causality: models, reasoning, and inference. Cambridge University Press New York, NY, 2nd edition.

[16] Judea Pearl. 2019. The seven tools of causal inference with reflections on machine learning. Communications of ACM, 62, 54-60.

[17] Judea Pearl and Dana Mackenzie. 2018. The book of why: the new science of cause and effect. Basic Books, New York, NY.

[18] Thomas J. Santner Brian J. Williams, and William I. Notz. 2003. The design and analysis of computer experiments. Springer, New York.

[19] Jennifer L. Skeem, Christopher Lowenkamp. 2016. Risk, race, and recidivism: predictive bias and disparate impact. Available at SSRN: https://ssrn.com/abstract $=2687339$

[20] Peter Spirtes, Clark Glymour and Richard Scheines. 2000. Causation, Prediction and Search. MIT Press, Cambridge, MA, 2nd edition.

[21] Caroline Uhler, Garvesh Raskutti, Peter Buhlmann, Bin Yu. 2013. Geometry of the faithfulness assumption in causal inference. Annals of Statistics, 41(2):436-463.

[22] Muhammad B. Zafar, Isabel Valera, Manuel G. Rodriguez, Krishna P. Gummadi Zafar. 2017. Fairness beyond disparate treatment and disparate impact: learning classification without disparate mistreatment. Proceedings of the 26th International World Wide Web Conference (WWW), 2017. pp. 1171-1180.

[23] Lu Zhang, Yongkai Wu, and Xintao Wu. 2017. A causal framework for discovering and removing direct and indirect discrimination. Proceedings of the Twenty-Sixth International foint Conference on Artificial Intelligence (IFCAI-17), pp. 3929-3935.

[24] Junzhe Zhang and Elias Bareinboim. 2018. Fairness in decision-making - the causal explanation formula. Proceedings of the AAAI Conference on Artificial Intelligence (AAAI-18), pp. 2037-2045.

[25] Junzhe Zhang and Elias Bareinboim. 2018. Equality of opportunity in classification: a causal approach. Advances in Neural Information Processing Systems, 2018. (NIPS 2018). 